\title{
Die Stadt tanzt! \\ Über den Prozess der Raumproduktion durch illegale Partys in Berlin
}

Schauplätze für informelle Praktiken sind der ständigen Transformation unterworfen. In Berlin sind vielfältige räumliche Manifestationen solcher Praktiken schon seit den 1970er Jahren bekannt. Darunter lassen sich unterschiedlichste Phänomene verstehen: von Subkulturen (zurück-)eroberte Freiräume über szenespezifische Treffpunkte bis hin zu Feiern unter freiem Himmel. Alle haben eines gemeinsam: Es geht um flüchtige, durch Konflikte geprägte, oft unsichere soziale Räume, die jedoch eine Chance auf gelebten Raum in der Stadt bergen. Hierin liegt das Interesse an einer kritischen Auseinandersetzung mit der räumlichen Entfaltung von informellen Praktiken, insbesondere von sogenannten illegalen Partys in Berlin.

Obwohl das Feiern auf unangemeldeten, illegalen Partys in den Sommermonaten regelmäßig vorkommt, ist das Phänomen nur sporadisch dokumentiert. In populären Medien sowie wissenschaftlichen Arbeiten (vgl. z. B. Lange 2007 und Golova 2011; beides Dissertationsschriften) tauchen Berichte über illegale Partys neben Ausführungen über andere informelle, szenebezogene Praktiken auf. Allerdings sind diese entweder knapp und unsystematisch oder der Fokus liegt eher auf etablierten Szeneräumen und klar identifizierbaren sozialen Gruppen. Illegale Partys sind verbreitet, aber ,unbekannt'. Dieses Paradox hängt vermutlich mit ihrem besonders flüchtigen und temporären Charakter zusammen. Die Veranstaltungen finden vorwiegend auf unscheinbaren Brach- beziehungsweise Restflächen statt und hinterlassen daher keine dauerhaft sichtbaren Spuren in der Stadt.

Nun stellt sich die Frage: Wie wirken sich illegale Partys im urbanen Kontext auf die Gesellschaft aus? Wir beziehen uns im Folgenden überwiegend auf die Techno-Szene in Berlin. Am Beispiel eines im Juli 2012 in Berlin-Treptow besuchten Events werden Veranstalter_innen, Gäste, Veranstaltungsort und Kommunikation beschrieben. Da es sich bei solchen Partys in der Regel um Veranstaltungen handelt, die gegen bestehende Regeln und Auflagen verstoßen, bildet die Rolle der staatlichen Kontrolle und die Interaktion mit der Polizei einen besonderen Schwerpunkt der Betrachtung. In Anlehnung an die marxistische Denktradition zu urbanen Räumen, insbesondere mit Bezug auf 
Henri Lefebvre, werden illegale Partys als Prozesse der Raumproduktion verstanden. Als analytischer Leitfaden dient hier die von Lefebvre (1991 [1974]) eingeführte Dreiheit der Dimensionen des Raums: die räumliche Praxis, die Repräsentation des Raums und der Raum der Repräsentation.

\section{Die ungeahnte Party}

Ein leises Brummen, ein Dröhnen am Sonntagnachmittag. Auf dem Weg in den Treptower Park scheint die Sonne. Der Park kommt näher, über die Brücke. Die Vermutung wird zur aufregenden Ahnung, der Bass wird lauter. Fahrräder anschließen, zwischen Brombeersträuchern und Pappeln hindurch zu den Menschen mit Bier in den Händen, ein Regen aus glitzerndem Konfetti. Unter der Brücke am Wasser ist die Musik laut, die Menschen bunt - Junge, Alte, Familien, Obdachlose. Eine improvisierte Bar, daneben ein DJ-Pult, davor leuchtende Buchstaben, eine Hängematte (vgl. Abb. 1).
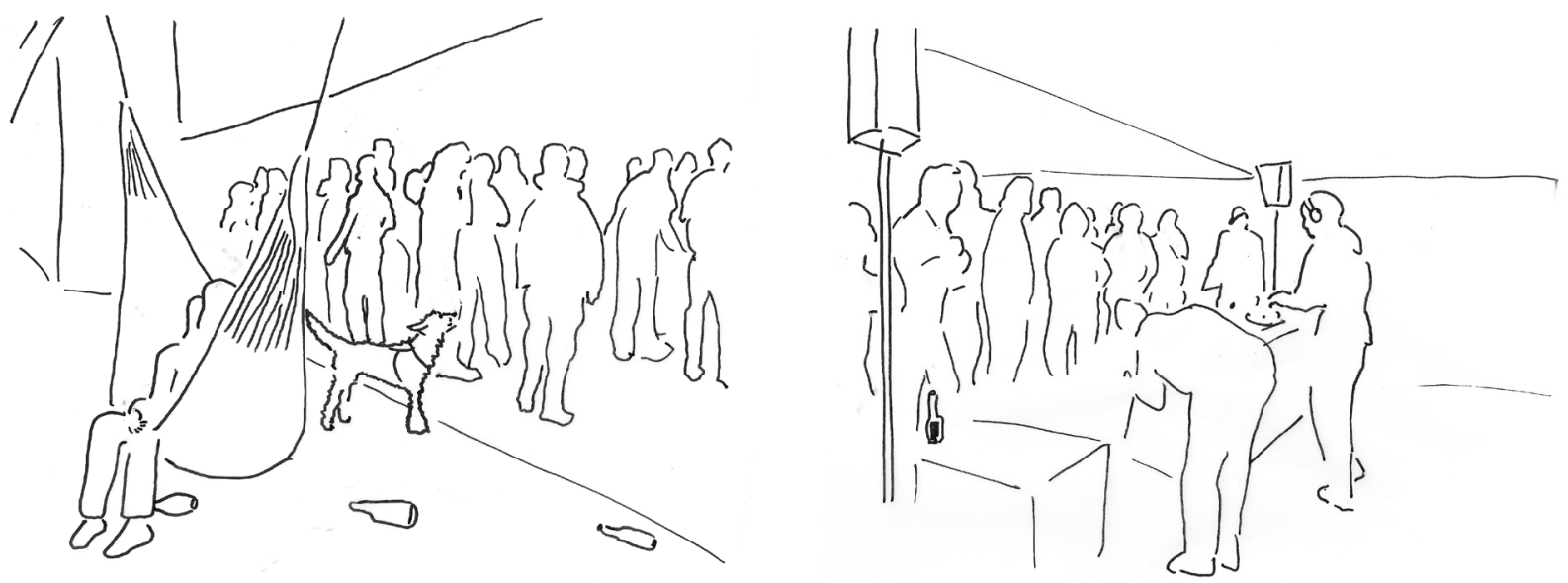

Die Anordnung der Körper im Raum, die Musik, die Art der Interaktion, die wenigen materiellen Objekten, die mitgebracht oder vor Ort zusammengestellt werden, tragen dazu bei, einen Prozess der Umdeutung des Raums für den Zuschauer sichtbar beziehungsweise erfahrbar zu machen (vgl. Abb. 2). Bezüglich der physischen und unmittelbar erfahrbaren Dimension des Raums kann von einer neuen räumlichen Praxis gesprochen werden. Es entsteht eine neue Nische des Feierns durch einen repetitiven, wenn auch unregelmäßigen Prozess, der das Potenzial des Ortes offenbart.

Abb. 1 Publikum (Quelle: Chantal Remmert, 2012)

Abb. 2 DJ-Pult und Tanzende (Quelle: Chantal Remmert, 2012)

Der Ort unter der Elsenbrücke in der Nähe des Treptower Parks scheint ideal für informelle Praktiken. Einerseits hat er eine visuell und akustisch unscheinbare Lage, in einer Vertiefung zwischen einer vielbefahrenen Straße und den S-Bahngleisen, andererseits ist der Ort stark frequentiert und gut erreichbar. Die Kultur der Aneignung kann in dieser architektonischen Grauzone nahezu ohne Aufsehen stattfinden.

Als illegale Party gilt in diesem Zusammenhang eine Veranstaltung auf öffentlich zugänglichem Gelände, dienichtbeim zuständigen Ordnungsamt angemeldet ist. Die Veranstalter_innen umgehen dadurch Auflagen der GEMA, die Zahlung einer Miete oder Pacht an den Besitzer des Geländes, die Beantragung einer Ausschankgenehmigung sowie eine Reihe von Bestimmungen zu Sanitäranlagen, eine Notausgangsbeschilderung oder ausreichend Sicherheitspersonal. Illegale Partys kosten keinen Eintritt und haben keine Türsteher_innen. Die Gäste können auch ihre eigenen 
Abb. 3 draußen sitzen (Quelle: Chantal Remmert, 2012)

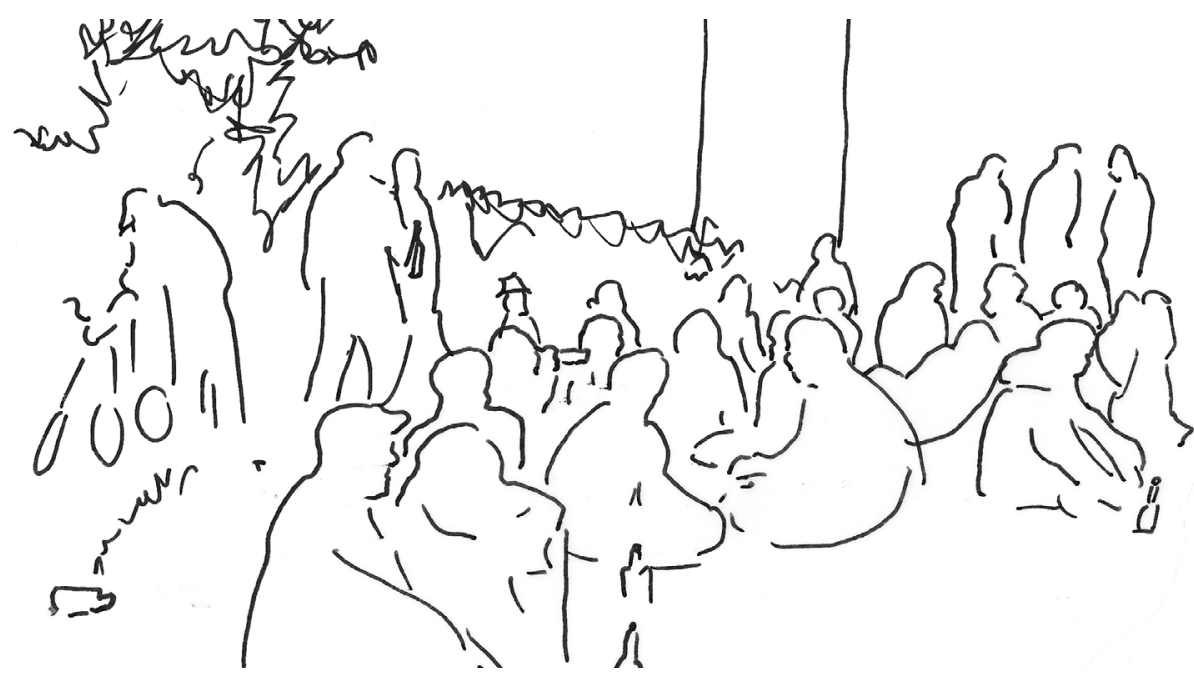

Getränke mitbringen. Meist aber werden Getränke verkauft, der Erlös wird in neues Equipment investiert oder zur Abzahlung von Strafen verwandt und kommt den Veranstalter_innen zugute.

Thematisiert wird hier das Spannungsverhältnis zwischen Tausch- und Gebrauchswert, ein Verhältnis, das den städtischen Raum mitprägt und durch den staatlichen Sicherheitsapparat aufrechterhalten wird. Durch ihren hauptsächlich unkommerziellen Charakter generieren illegale Partys einen neuen Gebrauchswert, der nicht vordergründig über Finanzielles definiert wird. Es bildet sich also ein Gegenpol zu den vom Tauschwert dominierten Alltagsräumen. Dies passiert spielend, besser gesagt: tanzend.

Die Besucher_innen dieser Partys sind großteils Techno-affine Menschen unterschiedlichsten Alters, viele jedoch sind zwischen 20 und 30 Jahren alt. Sie erscheinen als glitzernde Partygäste, welche die Afterhour genießen (vgl. Abb. 3). Anzutreffen sind ebenfalls Familien mit kleinen Kindern, die vermutlich zufällig am Ort des Geschehens vorbeigelaufen sind, die Musik und die Atmosphäre ansehen, mittanzen. Laut den Veranstalter_innen sind diese Partys für Freund_innen gedacht, sie werden über soziale Netzwerke wie Facebook benachrichtigt. Danach werden die Informationen über die Location und den Zeitpunkt der Party mündlich weitergegeben. Menschen, die zufällig auf sie stoßen, rufen oftmals von der Party aus ihre Freund_innen an.

Das einzige Ausschlusskriterium ist der fehlende Zugang zu sozialen Netzwerken wie Facebook. Den Veranstalter_innen kann ein Freundschaftsantrag gestellt werden, der von ihnen bestätigt werden muss. Dabei besteht die Möglichkeit zur Selektion. Bei anderen Internetportalen ist das Ausschlussverfahren härter: Menschen können sich nur anmelden, wenn ein Mitglied für sie bürgt. Jedes Mitglied kann nur einmalig für eine Person bürgen. Das bedeutet, es ist schwierig, gerade für Außenstehende, an diesem virtuellen Netzwerk teilzunehmen. Die angewendeten Kommunikationsformen und Auswahlmechanismen suggerieren, dass die Mitglieder von oben beschriebenen Internetportalen sich abschotten möchten. Durch die aktive Ausgrenzung der Gäste wird die Bewegung der illegalen Partys einerseits geschwächt. Anderseits verstärkt dieses Ausschlussverfahren das Wir-Gefühl, wirkt so identitätsstiftend und bietet Schutz vor (staatlicher) Kontrolle und Repression.

Die Veranstalter_innen nehmen das Risiko einer ungenehmigten Party bewusst in Kauf. Nach ihrer Auskunft gibt es meistens aber keinerlei 
negative Konsequenzen. Wenn sich Anwohner_innen beklagen - dies geschieht meistens ab 22.0o Uhr, aus Gründen der Lärmbelästigung -, wird in der Regel zusammengepackt und aufgeräumt. Hier unter der Brücke soll es möglich sein, lange ohne Beschwerden zu feiern (vgl. Abb. 4).

Um 22.56 Uhr erscheint die Polizei auf der untersuchten Party. In diesem Moment scheint es mehrere mögliche Szenarien zu geben: ein Tolerieren der Party, ein sanftes Beenden der Veranstaltung seitens der Veranstalter_innen und/ oder der Polizei oder eine harte und gewaltvolle Räumung des Raums durch den Sicherheitsapparat.

Der darauf folgende Ablauf scheint ruhig, routiniert und ernüchternd. Ungefähr acht Menschen in Uniform gehen zum DJ, danach verstummt die Musik, es wird zusammengeräumt. Die Besucher_innen gehen langsam weiter, einige bringen die leeren Flaschen zum improvisierten Tresen zurück. Der Platz wird von den Veranstalter_innen und den Besucher_innen aufgeräumt. Es scheint, als wäre die Musik und die Party nur eine Illusion gewesen.

Bemerkenswert an diesem Moment ist, dass sich nun in der bisher gemischten Menge von tanzenden Menschen Teilgruppen bilden: Die Organisator_innen geben sich als zuständige Ansprechpartner_innen zu erkennen, die Partygäste unterteilen sich in Interessierte und Sympathisant_innen, die beim Aufräumen helfen, während andere Besucher_innen sich zerstreuen, sobald die Polizei erscheint. Am Prozess der illegalen Partys ist scheinbar eine heterogene Gruppe an Besucher_innen beteiligt, und dies wird im Moment der Auflösung besonders deutlich. Diese Heterogenität ist teilweise auf die spontane, kurzfristige Organisation sowie die spezifischen Kommunikationsweisen zurückzuführen.

Über der Brücke ist die Polizei anzutreffen: acht bis zehn Uniformierte, mindestens einer in Zivil. Die Beamt_innen lehnen entspannt an den Polizeiautos, sie unterhalten sich gern mit uns, jedoch anfangs mit Zweifeln über die Ernsthaftigkeit des Gesprächs. Bereits die Körperhaltung der Beamt_innen zeigt ihre Überlegenheit sowohl in rechtlicher als auch in physischer Hinsicht. Im weiteren Ablauf sind erfahrungsgemäß kaum Auflehnungen gegen den Sicherheitsapparat zu erwarten. Die Praktik der illegalen Partys scheint zwar subversiv, jedoch nicht revolutionär zu sein.

Sehr gelassen wirkt ein älterer Polizist. Er erklärt, dass sie eine Party sofort nach dem Bemerken räumen müssten, auch wenn sich kein Mensch darüber beschwert habe. Er sagt, dass die Polizei weiterhin den Auftrag habe, sofort die Musikanlage in Beschlag zu nehmen, und dass eine Strafe von bis zu 50.00o Euro verhängt werden könne.

Abb. 4 Tanzende unter der Brücke (Quelle: Chantal Remmert, 2012)

Abb. 5 Eltern und Kinder (Quelle: Chantal Remmert, 2012)

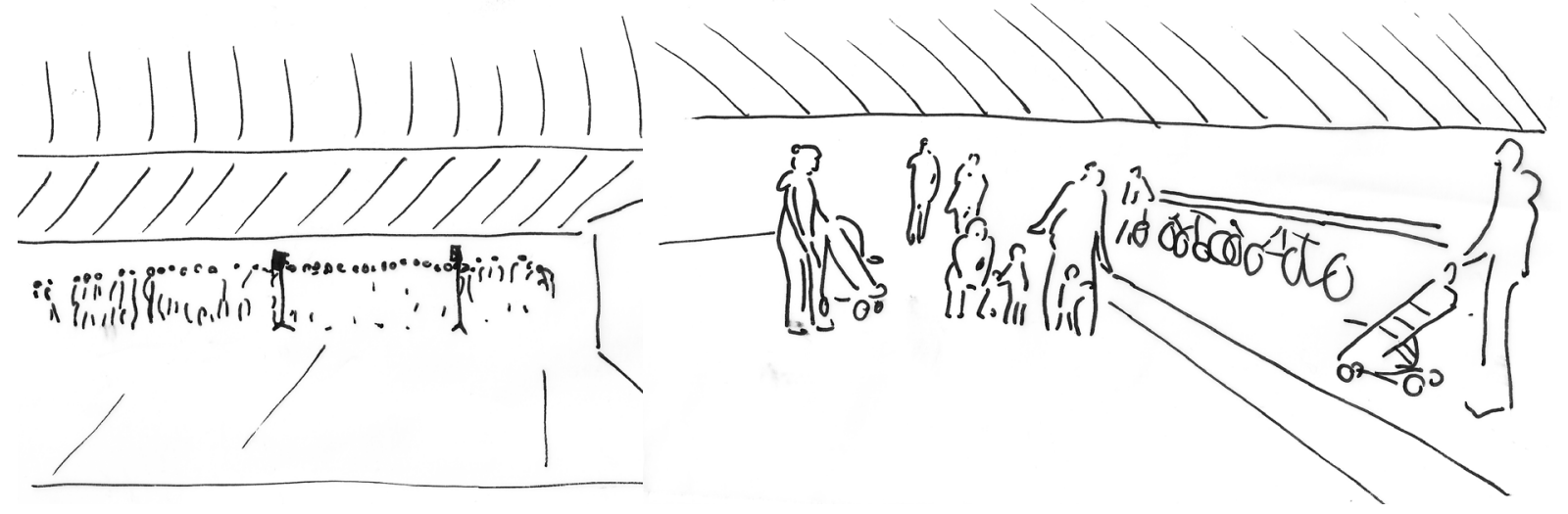


Die Organisation der Partys, die Durchführung und damit verbundene Neuinterpretation des Raumes, auch die Auflösung durch die Polizei, haben einen transformativen Effekt auf die Vorstellungen von dem Ort. Die bisher etablierte Ordnung des (konzipierten) Raums wird infrage gestellt: Ist die Party unter der Brücke ein szenespezifischer Treffpunkt, eine lokale Begegnungsmöglichkeit oder eine ordnungswidrige Tätigkeit? Die Zuordnung des Raums ist umstritten. Mit der Raumaneignung geht eine weitere Dimension, die neue Repräsentation des Raums, einher (vgl. Abb. 5).

Der urbane Raum der kapitalistischen Produktionsverhältnisse ist als homogen-fragmentiert zu verstehen (Lefebvre 1977) - homogen durch die Gleichsetzung aller Werte mit dem Tauschwert, fragmentiert durch die Eigentumsverhältnisse und Nutzungszuweisung. Orte der informellen Praktiken fallen aus diesem Muster heraus. Auch der Sicherheitsapparat operiert innerhalb dieses Paradoxes eines homogenen und zugleich fragmentierten Raums. Die Polizei als ausführende Staatsgewalt unterdrückt ,abweichende' oder ,gefährliche' Praktiken. Damit wird einerseits eine einheitliche Ordnung angestrebt, andererseits werden Trennlinien verstärkt.

Der informelle Charakter der initiierten Prozesse widerspricht der herrschenden Ordnung. Inwieweit äußert sich dieses subversive Potenzial? Es entsteht ein temporärer, flüchtiger Ort des Widerstandes. Um weiterhin stattfinden zu können, dürfen die nicht genehmigten Veranstaltungen keine dauerhaften Spuren im Raum hinterlassen. Aneignungsprozesse wie illegale Partys stellen einen Weg aus der Passivität der Nutzer_innen im homogenfragmentieren Raum dar. In diesem Zusammenhang kann man von einem gelebten Raum, einem gesellschaftlichen Raum der Repräsentation sprechen.

Als ob er dies unterbewusst spürte, erzählt der Polizist weiter, sie müssten Ordnungswidrigkeiten unterbinden. Ob sie dies dann auch täten, sei eine andere Sache. In der Praxis hänge es immer von der Gesprächsbereitschaft der Veranstalter_innen ab. Wenn diese nach mehrfachen Aufforderungen, die Veranstaltung zu beenden, immer noch nicht gehen wollten, gäbe es Probleme. Dies scheint jedoch die Ausnahme zu sein.

Illegale Partys bewegen sich an der Toleranzschwelle des Ordnungsapparats, von den Anwesenden werden sie in der Regel nicht als bedrohlich empfunden. Die Interaktion ist daher routiniert und wenig konfrontativ. Teil des gesamten Prozesses ist die Auflösung der Party durch die Polizei, die sich dadurch als wichtiger Akteur erweist.

Inzwischen sind alle gegangen, der komplette Raum ist gesäubert. Es wird gedankt, die Fahrräder werden aufgeschlossen, und es wird weitergefahren.

\section{Formalisierung informeller Praktiken}

Im Frühjahr 2013 eröffnete der Open-Air-Club ELSEan der Elsenbrücke. Ein Teil des Raums, auf dem früher die illegalen Partys stattfanden, wurde mit Holzverschlägen abgegrenzt. Die ELSE ist ein Ableger der „Wilden Renate“, einem Club, der sich über die Jahre auf der Straßenseite gegenüber etabliert hat. Laut den Betreiber_innen geht der Pachtvertrag über zehn Jahre, der Verpächter ist ein Privateigentümer. Somit ist aus der informellen Nutzung des Ortes eine formale und rechtlich legitimierte geworden. Bei regulären Veranstaltungen liegt der Eintrittspreis zwischen acht und zehn Euro (für 
Berlin typische Preise); manchmal finden auch sogenannte Umsonst-undDraußen-Open-Air-Veranstaltungen statt. Das vornehmlich sonntägliche Sommerprogramm war so erfolgreich, dass daran gearbeitet wird, den Club auch über die Wintermonate betreiben zu können.

Der Raum am Rand des Treptower Parks in Berlin beziehungsweise der jetzige Standort der ELSE ist ein klassisches Beispiel dafür, dass informelle Praktiken zu einer weiterentwickelten formalisierten Nutzung führen können. Dies hat zur Folge, dass die dort veranstalteten Partys nicht mehr spontan, kostenlos und wetterabhängig stattfinden, sondern über Wochen geplant und vermarktet werden. Die Restfläche verliert ihre Uneindeutigkeit, die als Nutzungsoffenheit und Einladung zur temporären Aneignung interpretiert wurde. Der Raum kann nun durch die kommerzielle Nutzung zu den homogen-fragmentierten Räumen gezählt werden; informelle Praktiken sind dort nicht mehr möglich.

Aus Türsteher_innen, Eintritts- und Getränkepreisen resultieren neue Verfahren zum Ausschluss von Besucher_innen, die dadurch auch eine zunehmend passive Rolle in der neuen Nutzungsform einnehmen. Mit der ELSE ist ein weiterer stabiler Treffpunkt für die Techno-Szene entstanden. Die aktuelle, formalisierte Nutzung wurde durch den Prozess der illegalen Partys ausgelöst und ersetzt nun die vorhergegangene, temporäre Nutzung.

Illegale Partys und ihre Verdrängungsprozesse verbildlichen den Kampf für und gegen eine Umdeutung und Neuordnung des städtischen Raums. Durch die Betrachtung des Phänomens können Diskussionen über den Wert und die Nutzung des Raums angestoßen werden. Illegale Partys in Berlin können ein temporärer Ausbruch aus dem kapitalistischen homogen-fragmentierten Raum sein, und mit ihren zum Teil subversiven Praktiken bergen sie auch ein Potenzial für utopische Denkanstöße.

\section{Autor_innen}

Chantal Remmert; Studium der Landschaftsarchitektur in Berlin; besonderes Interesse: Analyse und planerische Berücksichtigung dynamischer Prozesse im öffentlichen Raum chantal.remmert@gmx.de

Xenia Kokoula; Dipl.-Ing. Architektin; tätig in Forschung und Lehre an der Schnittstelle zwischen Landschaftsarchitektur und Städtebau

xenia.kokoula@mailbox.tu-berlin.de

\section{Literatur}

Golova, Tatiana (2011): Räume kollektiver Identität. Raumproduktion in der „linken Szene“ in Berlin. Bielefeld: Transcript Verlag.

Lange, Bastian (2006): Die Räume der Kreativszenen. Culturepreneurs und ihre Orte in Berlin. Bielefeld: Transcript Verlag.

Lefebvre, Henri (1977): Die Produktion des städtischen Raums. In: Arch+ 34, 52-57; gekürzt republiziert in: AnArchitektur 1/2002. https://wiki.zhdk.ch/vbk/lib/exe/ fetch.php?media=mittelbau:christian.fuerholz:aao1_lefebvre.pdf (letzter Zugriff am 10.8.2014).

Lefebvre, Henri (1991[1974]): The Production of Space. Oxford u. a.: Blackwell Publishers. 